\title{
HELMUT HENNE
}

\section{Jugendsprache und Jugendgespräche}

1. Ein jugendsprachlicher Anfang

2. Zum wissenschaf tspolitischen Rahmen

3. Zur Notwendigkeit der Erforschung der Jugendsprache

4. Zum Begriff 'Jugendsprache'

5. Jugendsprache in der Gruppe

6. Ein vorläufiges Fazit

\section{Erläuterungen* zu den Kapiteln 2 - 6:}

2. Angenommen wird eine Opposition von Theoriekonstruktion und Gegenstandsinteresse, wodurch die Absenz eines bedeutsamen Themas innerhalb der Linguistik des Deutschen erklärt wird.

3.1. Kindheit und Jugend als "Stadien auf des Lebens Weg" (Kierkegaard)

3.2. Die Nachzeichnung der sprachlichen Kindheit durch Spracherwerbstheorie und Kindersprachforschung und die Aufgabe, das sprachliche Stadium der Jugend nachzuzeichnen: Sprachprofilierung als Mittel zum Zweck

\subsection{Lexikalisierung der 'Jugendsprache' in der bisherigen Forschung}

3.4. Für einen sprachpragmatischen $Z$ ugang zur Jugendsprache: J u g e n d g e s p r ä che, Jugendbriefe und -literatur als empirische Basis

4.1. 'Jugendsprache' als $\mathrm{s} p$ e $\mathrm{z}$ i f is $\mathrm{c}$ h e Sprech- und Schreibweisen, mit denen Jugendliche ihre Sprachprofilierung betreiben; Sprachprofilierung als Mittel der Identifikation und Identitätsfindung

4.2. Sprachliche Profilierung und deren individueller und kollektiver Aspekt; standardsprachliche und umgangssprachliche Kommunikation; Sprachprofilierung in der Gruppe: der 'Jugendton' als sprachliches Ergebnis der Kommunikation in Peer-Groups, Cliquen, Freundeskreisen, Banden; deren Funktion: Abgrenzung nach außen ("Ihr"), Identifikation nach innen ("Wir"): "Und sie suchen Freundschaft und Kameradschaft mehr als andere Lebensalter, weil sie am Zusammenleben Freude haben [...]" (Aristoteles)

4.3. Zur sozialen, regionalen, alters- und geschlech tsspezifischen Differenzierung von Jugendsprache

4.4. Insofern die Gruppe Kristallisationspunkt jugendsprachlicher Profilierung ist, sind $\mathrm{J} u \mathrm{~g}$ e n d g e sprä che der n o t we ndig e Ausgangspunkt jugendsprachlicher Forschung.

4.5. 'Jugendkultur' als Sub-, Teil oder Gegenkultur? Die Begriffe einer Vorbildbzw. Echo-Sprache 
5. Jugendsprache in der Gruppe

5.1. Am Beispiel des Grüßens, der Anrede und Erwiderung

5.2. Am Beispiel von Gesprächspartikeln

5.3. Am Beispiel des Sprechcharakters jugendlicher Schreibe

6. Jugendsprache in der Gruppe ist experimentell, antikonventionell und tendenziell situationalisierend. Sprachkreativität ist das Vehikel dieser Eigenschaften, wobei Metaphorisierung und Hyperbolisierung die auffälligsten Züge sind. - Jugendsprachforschung und kritische Sprachwissenschaft

\section{Ein jugendsprachlicher Anfang einer standardsprachlichen Rede}

Meine Damen und Herren, schon längst bin ich auf der Suche nach einem neuen Anfang - zumindest dem eines wissenschaftlichen Vortrags. Hier ist der neue Anfang - jugendsprachlich:

Tussis und Gents, Fans alle zusammen! Hey Freaks! Ich weiß, alle seid ihr auf der Suche nach der großen heißen Message. Logo, die einen gehen auf den Theorie-Trip, die andern törnen sich mit Fakten an und alle seid ihr echt high, wenn ihr euch gegenseitig Wissenschafts-Chaoten nennt und euch beknackte Buschwedeleien vorwerfen könnt.

Meine Damen und Herren, wenn ich in diesem Stil weitermachen sollte, wäre das, möglicherweise, ganz unterhaltsam; aber die heiße w i s s e n $\mathrm{s} \mathrm{ch}$ a f $\mathrm{t} \mathrm{l}$ i c h e "Message", hinter der auch ich her bin, ist nur standard- und wissenschaftssprachlich zu haben, die heiße Message 'Jugendsprache' nämlich.

\section{Zum wissenschaftspolitischen Rahmen}

Indem ich nun zu Beginn, per Imitation, den folkloristischen Aspekt des Themas herausstelle, stehe ich in der Gefahr, den Ernst und die Würde meines Themas zu verraten. Um diesen Ernst und diese Würde geht es mir nämlich vor allem.

Ich möchte hier die These vertreten, daß die germanistische Linguistik, also die Wissenschaft von deutscher Sprache, sich zu lange und zu ausschließlich von Fragen der Theorie- und Methoden $\mathrm{k}$ o $\mathrm{n} \mathrm{st} \mathrm{r} u \mathrm{kt}$ i o $\mathrm{n}$ hat leiten lassen. Entwicklungen der Sprachgermanistik stellen sich dar als Theorieablösungen und -modifikationen, die durch Defekte der jeweils herrschenden Theorie bedingt sind. Dazu nur ein Beispiel von vielen: Die Intensität, mit der auch hierzulande Spracherwerbsforschung betrieben wurde, rührt wesentlich daher, daß Chomsky dieser Forschung die generativistische Weihe gab; indem er nämlich die "Erklärungsadäquatheit" einer linguistischen Theorie an "die Frage nach der Konstruk- 
tion (sic) einer Theorie der Spracherlernung" band. ${ }^{1}$ Jede e in z e l $\mathrm{s} \mathrm{p} \mathrm{ra} \mathrm{ch}$ li $\mathrm{c}$ h e Linguistik hat aber Themenbereiche zu bearbeiten, die sich nicht in das jeweils herrschende Theoriekonzept einer a $1 \mathrm{lg} \mathrm{e} \mathrm{m} \mathrm{e} \mathrm{i} \mathrm{-}$ $\mathrm{n}$ e $\mathrm{n}$ Linguistik fügen. Solche Themenbereiche sind sprachpolitisch z.T. unabweisbar und stellen, populär gesprochen, eine Herausforderung an die Sprachforscher dar.

Nur wenn man in dieser Weise akzentuiert, wird es verständlich, daß das Thema Jugendsprache bis heute nicht zu einem ernsthaften Thema germanistischer Sprachforschung wurde, obwohl gerade seit 1960 Entwicklungen eingetreten sind, die das Thema als germanistisches unabweisbar machen.

\section{Zur Notwendigkeit der Erforschung der Jugendsprache}

Kindheit und Jugend, so viel weiß man schon seit den Anfängen der Pädagogik in der griechischen Antike, sind für das Leben des Einzelnen entscheidende "Stadien auf des Lebens Weg" (Kierkegaard) ${ }^{2}$. Die Sprachwissenschaft hat dem insofern Rechnung getragen, als die Erforschung der Sprache und der Sprachentwicklung der 0 bis 12 jährigen zu einer wichtigen Domäne moderner Linguistik geworden ist. ${ }^{3}$ Sie hat dem in keiner Weise Rechnung getragen insofern, als es eine linguistische J u g e n dsprachforschung nicht gibt. Demzufolge auch keine $\mathrm{g}$ e $\mathrm{r} \mathrm{m}$ a $\mathrm{n}$ is $\mathrm{t} \mathrm{i}$ $\mathrm{s} \mathrm{c}$ h e Jugendsprachforschung. Wenn das Stadium der Kindheit als das des Spracherwerbs gelten kann, so möchte ich das Stadium der Jugend, das von 13 ab zu rechnen ist und ein variables Ende hat, als das der "Sprachprofilierung" bezeichnen. Wenn der Erwerb von Sprache die Hauptleistung der Kindheit ist, so ist der Erwerb einer an der Erwachsenenwelt orientierten Identität die (nicht immer erreichte) Hauptleistung des Jugendalters. Erwerb von Identität ("die als Selbst erlebte innere Einheit einer Person") aber bedeutet auch: relativ zur kulturellen Umgebung einen eigenen Sprachstil zu finden. Der Einstieg zu einem eigenen Sprachstil erfolgt nun vielfach über eine Gruppe, die "U n s " und unsere Gruppe von " $E$ u $\mathbf{c}$ h ", den Erwachsenen, abhebt. W i $r$ - Gefuihl als eine Form der Identifikation, also der Ubernahme der Motive und Eigenschaften anderer in das eigene Ich, und, daraus resultierend, I h $\mathrm{r}$ - Entfernung sind Etappen auf dieser Identitätsbildung. Über Identifikation und Abgrenzung führt der Weg zur Identität und damit zu einem eigenen sprachlichen Profil. ${ }^{4}$

Wenn nun von sprachwissenschaftlichen Beiträgen zur Jugendsprache zu sprechen ist, so von solchen, die das Thema Jugendsprache erst einmal lexikalisieren und bisweilen in der Gefahr stehen, den jugendsprachlichen Wortschatz auf das Niveau von Exotismen zu drücken. $\mathrm{Zu}$ nennen sind hier 
die Arbeiten u.a. von G. Heinrich, S.A. Wolf, H. Markus, Sabine Pape, H. Küpper und Lorelies Ortner. ${ }^{5}$ Um nicht mißverstanden zu werden: Ich schelte nicht die Verfasser dieser Beiträge, die vielmehr wichtige $V$ o $r$ arbeiten zur Jugendsprache darstellen; aber ich muß doch darauf bestehen, daß das Thema 'deutsche Jugendsprache' ein sprachpragmatisches Theoriekonzept erfordert. Die empirische Basis eines solchen Konzepts müssen dann Jugendgespräche, Jugendbriefe und Jugendliteratur, also Tagebücher und ähnliches sein. Und an dieser Stelle wird der $\mathrm{g}$ e $\mathrm{r} \mathrm{m}$ a $\mathrm{n}$ is $\mathrm{t}$ is $\mathrm{ch}$ e Nutzen der Gesprächsforschung unmittelbar einsichtig. Es geht in $\mathrm{n}$ e $\mathrm{rh}$ a l b der Germa $\mathrm{n}$ is $\mathrm{t}$ ik nicht um eine Dialogforschung als solche, sondern um eine Gesprächsforschung, die sich in den Dienst einer deutschen Textlehre stellt und u.a. dialogische Textstrukturen und -elemente erarbeitet, aus der jugendlichen Gesprächssprache z.B. deren Gesprächsstrukturen und Versatzstücke, Gesprächswörter, Sprecheigentümlichkeiten, Wortschätze und Wortbildungsmittel.

\section{Zum Begriff 'Jugendsprache'}

Bevor ich nun weiter ausführe, wie Jugendsprachforschung und Gesprächsforschung zusammengehen, muß ich den Begriff 'Jugendsprache' weiter präzisieren. 'Jugendsprache' bezeichnet $\mathrm{s} p \mathrm{e} z$ if is $\mathrm{ch}$ e Sprech- und Schreibweisen, mit denen Jugendliche u.a. ihre Sprachprofilierung und damit ein Stück Identitätsfindung betreiben. E i n e der Möglichkeiten dieser Profilierung besteht nun darin, einen eigenen "Jugendton" in der jugendlichen Gr u p p e zu pflegen. Diese Form sprachlicher Profilierung ist so gängig und üblich, daß man dazu neigt, sie als einzige Form einer spezifischen jugendlichen Sprechweise zuzulassen. Hinzuweisen ist aber darauf, daß Sprachprofilierung in der Gruppe nur der kollektive Aspekt der Identitätsfindung oder besser: Identitätssuche ist, die ein individuelles Ergebnis haben muß und insofern auch ausschließlich individuell betrieben werden kann.

In der Jugendsoziologie gibt es für die Gruppenorientierung Jugendlicher den Begriff der "Peerbeziehungen". 6 'Peer-group' ist ein der amerikanischen Soziologie entlehnter Begriff ${ }^{7}$ und meint die annähernd gleichaltrige Bezugsgruppe Jugendlicher, die eine gleiche oder ähnliche Interessenlage und soziale Herkunft haben. In der Jugendsoziologie hat man nun festgestellt, daß seit den 60er Jahren die Gruppenorientierung der Jugendlichen zunehmend intensiver wird und entsprechend die familiale Autorität zurücktritt. ${ }^{8}$ Im Zusammenhang damit ist dann die Etablierung einer spezifischen Jugendkultur zu verzeichnen, von der 
nicht sicher ist, ob sie eine Gegenkultur, eine Subkultur oder eine mit der Gesamtkultur verträgliche Teilkultur darstellt. Zwar wird die Wortzusammensetzung Jugendkultur noch nicht in den großen Wörterbüchern der deutschen Gegenwartssprache in Ost und West geführt ${ }^{9}$, aber Begriff und Wort waren bereits zu Anfang des 20. Jhs. programmatisch und haben sich längst durchgesetzt. ${ }^{10}$ Dafür sorgen schon die Medien und der Kommerz, die fortlaufend das verbreiten, was sie für Jugendkultur halten. 'Jugendkultur' hat, unabhängig von der Vermarktung, drei wesentliche Eigenschaften: sie ist $f$ ü $r$ Jugendliche $v$ o n Jugendlichen entworfen erstens; sie konstituiert sich durch die "psychische Bindung" Jugendlicher an Gleichaltrige - zweitens; und sie tendiert zur Autonomie in n e r h a l b der Erwachsenenkultur - drittens. ${ }^{11}$ Das hat natürlich sprachliche Folgen. Welche - darüber gibt es bis jetzt nur spärliche, höchstens lexikalische Antworten. Insofern nun die jugendliche Gruppe, die Peer-Group in der Form des Freundeskreises, der Clique oder Bande, Kristallisationspunkt jugendsprachlicher Profilierung ist, sind $\mathrm{J}$ u g e n d g e s p r ä c h e der notwendige methodische Ausgangspunkt jugendsprachlicher Forschung. Im G e s p r ä c h der Peer-Group erhält Jugendsprache einen kreativistischen Grundzug, der in erster Linie i $m$ Gespräch auffindbar und deshalb innerhalb einer Gesprächsanalyse beschreibbar ist. Diese sprachpragmatische Ausrichtung der Forschung erhält nun eine zusätzliche sprachsoziologische Dimension insofern, als die peer-group-orientierte Jugendsprache regional, sozial, alters- und geschlechtsspezifisch differenziert ist - wenn man den Begriff 'Peer-Group' ernst nimmt, könnte man sagen: per definitionem. Diese komplexen Strukturen werden nun insofern noch unübersichtlicher, als jeweils mit einer Vorbild- und Echo-Sprache in den Medien zu rechnen ist. Vorbild-Sprache: Es gibt Idole der Jugendlichen, deren sprachliche Allüren Eingang in ihre Gruppensprache finden, und überraschenderweise können das auch literarische Idole sein. Die Existenz des jugendsprachlichen Grußes Ave zum Beispiel hat keinen religiösen Hintergrund ("Ave-Maria"), sondern geht auf jenen "listigen kleinen Krieger voll sprühender Intelligenz" zurück, der ein Gallier ist, den Namen Asterix trägt und den Römern hinhaltenden Widerstand leistet, wodurch dann auch römische Grüße, eben $A v e$, in die SprechblasenGespräche kommen u n d von Jugendlichen übernommen werden: Die Comic-Serie "Asterix und Obelix" nimmt, eben wegen ihres Sprachwitzes, eine Vorbild-Funktion ein. Echo-Sprache: Das ist die Sprache der Redakteure von Jugendzeitschriften und Moderatoren jugendlicher Rundfunksendungen, die sich mit den Jugendlichen verständigen wollen und müssen und dabei auf deren, vielleicht vermeintliche, Sprache eingehen. Am 5.10.1979, ca. 13.40 Uhr, sagt der Moderator der NDR 2-Sendung "Musik 
für junge Leute": "Anyway, jetzt geht's los!" Ist anyway eine neue gesprächsakt- oder gesprächsschritteinleitende Partikel der Jugendsprache, die eine Chance hat, als Anglizismus, wie Sound oder Disco oder Freak, in die Standardsprache übernommen zu werden, oder ist das sprachliche Anbiederung, die nichts so sehr fürchtet wie die normale "Autoritätssprache" der Erwachsenen?

\section{Jugendsprache in der Gruppe}

\subsection{Am Beispiel des Grüßens, der Anrede und Erwiderung}

Die Autoritätssprache der Erwachsenen kommt spätestens zu Anfang dieses Jahrhunderts unter jugendlichen Druck. Die "Jugendbewegung" am Anfang des 20. Jhs., die sich selbst z.B. den Namen 'Wandervogel' gab, pflegte eine Sprache, die sich u.a. der studentischen Burschensprache und mittelalterlichem Vagantentum anzulehnen versuchte. Ähnliche Vorbilder, literarisch aufgeputzt, läßt der folgende Text erkennen, der dem Roman von F. Torberg, Der Schüler Gerber hat absolviert. Berlin [usw.] 1930, $251 \mathrm{f}$. entnommen ist:

"He, Unlustknabe!"

Jemand berührte seine Schulter. Kurt schrak zusammen, als hätte man ihm einen Revolver vorgehalten. Dann blickte er auf und erkannte Boby Urban.

"Daß dich der gelbe Geier hacke! Was rauschest du mit hochgesenktem Haupt an mir vorüber?"

Der gelbe Geier, Wieso. Es gibt keinen gelben Geier. "Guten Tag." Kurt ergriff die dargebotene Hand.

"Na endlich" lachte Boby Urban. ...

Da lacht jemand. Woher kommt dieser da, welcher lacht. Er lacht noch immer. Da muß ich wohl auch lachen. Haha. Es wird schon gehen.

Der antike Lustknabe wird hier sprachspielerisch zum Unlustknaben verkehrt - schon hier eine binäre Obsession, die später die Linguistik befallen wird; das gesenkte Haupt wird zum bochgesenkten Haupt: Die rhetorische Figur des Oxymorons, in der einander sich widersprechende Bedeutungskomponenten zusammengefügt werden ('hoch' und 'gesenkt'), gibt dem Textstück etwas Übertreibend-Dramatisches, das durch das Vorüberrauscben in altertümlich-poetischer Grammatik ("Was rauschest du [...]") noch verstärkt wird. Und auch der alliterierende "gelbe Geier" ist ein literarisches Geschöpf: Zum ersten Mal von Gryphius in verhüllender Manier für den 'Teufel' gebraucht ${ }^{12}$, ist der Gelbe Geier hier ein Verwünschungstopos, der gute Laune signalisieren 
u n d Gymnasiasten-Erkennungsmelodie zugleich sein soll. Aber nicht nur der literarisierte Stil stempelt diese Szene zur jugendlichen $\mathrm{G} \mathrm{y} \mathrm{m} \mathrm{n}$ a $\mathrm{s}$ i a $\mathrm{s}$ t e $\mathrm{n}$ sprache, auch die Form der Gesprächseröffnung ist spezifisch jugendsprachlich. Aus dem mir zur Verfügung stehenden Material "Zum Grußverhalten Jugendlicher in der Gegenwart" 13 ist ersichtlich, daß 2.B. 15 - 16jährige nichts so sehr meiden, ja man kann sagen fürchten, wie die konventionellen Erwachsenengrüße - zumindest wenn sie sich selbst begrüßen. Die Jugendgrüße lauten z.B.: Hallo, Tacbchen, Salut, Hi, wie siebt's aus, ballo friends, Grüß Göttle, n'wunderschönen, na du Bär, na du Eimer, bey Freaks. Wollte man aus dem mir zur Verfügung stehenden Material eine Normalform herausfiltern, wäre das möglicherweise Hallo, wollte man eine Tendenzform herausarbeiten, wären das Phrasen mit na.

Der Beginn des österreichischen Gymnasiastengesprächs von 1930 ist aber nicht nur wegen seiner im Vergleich zur Standardsprache entkonventionalisierten Begrüßungsformel (He Unlustknabe) tendenzweisend; insofern dieser Gruß als symbolisch vermittelter Beginn eines Gesprächs mit einer nichtsprachlichen Körpersymbolik verbunden ist ("Jemand berührte seine Schulter [...]"), ist er gleichfalls für jugendsprachliche Grüße, die ein Gespräch eröffnen, typisch; denn zu unterscheiden sind "Grüße im Vorbeigehn" einerseits und "Uberraschungsbegrüßungen" und "Verabredungsbegrüßungen" andererseits. ${ }^{14}$ Erstere scheinen als Sprachformen kaum eine Rolle in der Jugendsprache zu spielen, letztere die eigentlich interessanten u n d gehätschelten sprachlichen Wendungen zu sein. In ihrer Form unmittelbarer Anrede, Typus: $N a, d u B a ̈ r$, sind sie distanzüberwindend, vertrauensschaffend und zur Erwiderung auffordernd. Sie besitzen eine unmittelbare Kraft, den Partner in das Gespräch zu ziehen.

Der Gesprächs r e a l i s m u s des $\mathrm{f}$ i $\mathrm{k} t$ i o n a l e n Gymnasiastendialogs vermag in seiner pointierten Treffsicherheit zuweilen gesprächsanalytische Definitionen, etwa folgenden Typs, zu erweitern: "Ublicherweise wird ein Gespräch mit einem gegenseitigen Gruß eröffnet" ${ }^{15}$; dieser Dialog übertrifft auch Formulierungen, die kundtun, daß es sich bei der Dialogkonstituierung "im wesentlichen um ritualisierte und verfestigte Sprachelemente" handele ${ }^{16}$ : Weder liegen in unserem Fall eine gegenseitige Grußsequenz noch unbedingt verfestigte Sprachelemente vor. Vielmehr liegt das vor, was in traditionellen Gesprächsanalysen immer wieder zu kurz kommt: eine Form v o r lä u f i g e r Gesprächsverweigerung. Der Grund dafür, daß diese Kategorie bisher wenig entwickelt ist, liegt u.a. darin, daß Pausen nur als Pausen, symbolisiert z.B. durch Kreuze (+), erscheinen. ${ }^{17}$ Das heißt: Auch die besten gesprächsanalytischen Notate präsentieren nur die sprachliche Oberfläche, die sprachliche Seelenarbeit kommt nicht in den Blick der Analytiker. Dazu muß man nicht nur auf Jugendgespräche 
zurückgreifen, sondern auch auf Jugendgespräche in der Literatur: Nach der 'Überraschungsbegrüßung' verweigert sich der Gesprächspartner zunächst; das führt den Anredenden dazu, dem Gesprächspartner einen Gruß zur Gesprächserwiderung zu entbieten ("Daß dich der gelbe Geier hacke!") u n d zugleich eine 'Nachfrage' nachzuschieben ("Was rauschest du mit hochgesenktem Haupt an mir vorüber?"), die eine dritte inhaltlich gefüllte Form der Anrede und damit eine zweite Aufforderung zur Gesprächserwiderung darstellt. Somit besteht der erste Gesprächsschritt des Boby Urban aus drei Gesprächsakten, die jeweils als "minimal-kommunikative Gesprächseinheiten" 18 einen handlungsplanmäßigen Stellenwert haben: Ich möchte diese Gesprächseröffnungsstruktur, die eine dreifache Steigerung darstellt, eine 'Anrede-Klimax' nennen. In sie verwoben ist die sprachliche und nicht an die Oberfläche kommende Seelenarbeit des Angeredeten, wofür die Literaturwissenschaft u.a. den Begriff eines 'inneren Monologs' kennt: "in Romanen und Erzählungen die Wiedergabe von in Wirklichkeit unausgesprochenen Gedanken, Assoziationen, Ahnungen [...] in Sprache direkter Ich-Form [...]". ${ }^{19}$ Bei näherem Zusehen erweist sich, daß die "unausgesprochenen Gedanken" zumeist keinen 'inneren Monolog', sondern einen 'inneren Dialog' insofern darstellen, als sie auf ein Gegenüber ausgerichtet sind und ihre besondere Ausprägung eben durch die Ausrichtung am Gesprächsschritt eines anderen erhalten.

Und eine weitere Lehre gibt die gymnasialsprachliche Gesprächseröffnung aus dem Jahr 1930 auf: daß nämlich jugendsprachliche Gespräche standardsprachlich versanden können. Der 'Jugendton' wird in diesem Fall von Kurt Gerber verweigert - aus Gründen innerer und äußerer Disposition. Am Ende der Jugend steht der Abschied von der Jugend(sprache); dieser Abschied wird vorbereitet in jugendsprachlichen Verweigerungen, hier indiziert durch das standardsprachliche "Guten Tag".

\subsection{Am Beispiel von Gesprächspartikeln}

Die literarisch besetzte Jugendsprache der 30er Jahre unseres Jahrhunderts ist eine Sache; die andere ist die, welche in den wilden Peer-Groups unserer Tage gepflegt wird. So viel ist inzwischen von dieser Sprache bekannt, daß sie zu satirischen $Z$ wecken eingesetzt werden kann, etwa wenn in Anlehnung an die 'Sonthofener Rede' von F.J. Strauß Helmut Schmidt eine Rede im Jugendton in den Mund gelegt wird, eine Rede, die er - natürlich - in der Disco "Sponti-Lollipop" in Dingswede am Steinhuder Meer gehalten haben soll. ${ }^{20}$ In solchen Satiren erscheinen, z.T. kunstvoll gehäuft, jugendsprachliche Versatzstücke, aber nicht d i e Jugendsprache. Dazu beherrschen die Satiriker kaum oder zu wenig die Partikel-Welt der Jugendsprache. Hier artikuliert sich nicht nur 
die Stilnot der weniger eloquenten Jugend; sondern auch das in seiner Schriftferne solidarische Sprechen, das den anderen in den eigenen Gesprächskreis zieht und ihn mit Partikeln umgarnt, so wie in dem folgenden Ausschnitt aus einem Interview ${ }^{21}$ :

M.: Wir Auszubildenden untereinander duzen uns also erstma generell, also auch hier im Betrieb. Naja und die Mitarbeiter, die also ausgelernt haben, werden also in der Regel gesiezt, solange bis einem das Du eben angeboten wird. Oder wenn man merkt, der is älter, dann bietet man selbst das Du an, naja wenn man mit den Leuten eben so zusammenarbeitet. Naja ...

H. : ... und ich laß mir lieber das Du anbieten, ne, das war ja al* ich bin ja auch hier, also früher war das ja nich so. Inner Schule, da hat man halt geduzt ...

Die Partikelforschung ist ja (sic) kein Ruhmesblatt der Germanistik. Sie konnte allerdings erst reussieren, als der dialogische Aspekt gesprochener Sprache in den Blick kam. In bezug auf die Steuerung des Hörers durch den Sprecher liegen, gesehen z.B. auf das naja, strukturierende Gesprächsakte vor, durch die der Sprecher seinen Beitrag gliedern und damit auf den Hörer entsprechend seinen Intentionen einwirken will. ${ }^{22}$ Insgesamt liegt also eine "Hörersteuerung" durch den Sprecher vor, die Jochen Rehbein in "Vorschaltungen", hier z.B. naja, und "Nachschaltungen", hier z.B. ne, unterteilt und dabei, dem binären Schema verhaftet, die 'Zwischenschaltung' vergißt. ${ }^{23}$ In dem hier vorliegenden Gesprächsfragment ist das naja eher als Zwischenschaltung zu begreifen, weil es nicht einen Gesprächsschritt einleitet, sondern $\mathrm{z}$ w is c h e $\mathrm{n}$ den Gesprächsakten der Sprecherin vermittelt und insofern den Hörer steuert. Insgesamt nennt Rehbein die Vor- und Nachschaltungen "Augmente" insofern, als die sprachliche Handlung 'erweitert' sei dadurch, "daß [zusätzliche] sprachliche Elemente die kommunikative Funktion der Hörersteuerung übernehmen" 24. Die Kategorie 'Augment' (also "Vermehrung") nähert sich dabei bedenklich der normativen Einschätzung jener Stilistiken, die diese Gelenkstellen am Körper des Gesprächs als Füll- oder Würzwörter abtaten; demgegenüber ist festzuhalten: strukturierende und appellierende Gesprächsakte sind Konstitutiva des Gesprächs. Mit dem ersten naja des Texts konstituiert die Sprecherin einen Gegensatz (und damit einen Zusammenhang) zwischen den ersten beiden Gesprächsakten ("Wir Auszubildenden [...] generell, also [...] Betrieb") einerseits und der folgenden Rede andererseits und appelliert damit zugleich an den Hörer, dieser Darstellung zu folgen gemäß dem Motto: "Du weißt schon, wie das läuft ...". Wollten Sie, meine Damen und Herren, die von mir eingestandene Subjektivität meiner Interpretation von 
naja überprüfen, möchte ich Ihnen raten, nicht zu unseren großen Wörterbüchern der deutschen Gegenwartssprache zu greifen, die naja nicht oder nur als "Ausruf der Zustimmung" im Sinne von Naja, endlich, also: "da haben wir's" führen. ${ }^{25} \mathrm{Naja}$, so könnte man pointiert sagen, hat hier, gesehen auf die Semantik des Gesprächsschritts insgesamt, eine verkürzende und nicht eine 'augmentierende' Funktion. Die Gesprächssemantik von naja müßte nämlich in einem schriftlichen Text in eine längere Phrase überführt werden.

Jugendsprachliche Gesprächstexte, das ist nun meine These, leben von solchen Partikeln, die ihre Semantik erst in der j e w e il i g e n Gesprächssituation entfalten und deshalb nur mäßig jenseits und außerhalb dieser Gesprächssituation beschrieben werden können.

\subsection{Am Beispiel des Sprechcharakters jugendlicher Schreibe}

Während schriftliche Texte, zumindest fachlicher Art, eine jeweils vergleichbare kommunikative Wirkung gerade außerhalb spezifischer Kommunikationssituationen entfalten, sind alltägliche Gesprächstexte erst einmal an die jeweilige Situation, an die g e m e i n s a m e Umweltinterpretation und -orientierung und die daraus resultierenden gemeinsamen Vorannahmen gebunden. Jugendsprache in der Gruppe verstärkt nun diesen gesprächssituationellen Charakter ihrer Sprache, indem sie z.B. Grüße und Anrede entkonventionalisiert und gesprächsspezifische Partikeln gehäuft verwendet, damit aber eine an der Schriftsprache orientierte Erwachsenengeneration (bewußt?) irritiert. Nicht genug damit, zersetzt sie, partikelwütig, auch noch fachliche schriftliche Texte, z.B. diesen 26 :

Atemlos hocken die Fans da und Lou gibt seinem Rock-Herzen den gar nicht mehr für möglich gehaltenen Zusatzkick [...] Und hier hört der Film auf: Jeder muß jetzt für sich selbst diese Scheibe gut finden.

Wenn nicht? ? Hm, hm ... Äh, hm - ach, den Fall gibt's doch gar nicht. Oder?

Dieser Text (recte: dessen Verfasser) verunsichert insofern, als er mit Verlegenheitspartikeln $(\mathrm{bm})$, Pausenfüllern $(\ddot{a} b)$ und Gliederungspartikeln (oder) eines imaginären Sprechers (als Antwortendem) angereichert ist, obwohl es für den zitierten Text gar keinen Sprecher und Hörer, sondern nur einen Schreiber und Leser gibt. Die Funktion dieser Gesprächspartikeln in der geschriebenen Kommunikation besteht darin, diese stilistisch anzureichern, die Gesprächspartikeln also als Stilmittel in der "Schreibe" einzusetzen. Der Schallplattenrezensent möchte seinen Text dialogisieren und Einwände gegen seine Bewertung auf das Maß hilfloser Gesprächssprache drücken - in durchaus ironischer Manier. In einem Leserbrief an die Musikzeitschrift "Sounds" schreibt ein Fan aus Bonn: "Zum schluß noch 
billige anbiederung meinerseits - ihr habt euch - ähm - ganz proper entwickelt." 27 An dieser Stelle hätte ein traditioneller erwachsener Schreiber einen Gedankenstrich gesetzt ("Ihr habt euch Gedankenstrich ganz proper entwickelt!"), um die eigentliche Aussage ("ganz proper entwickelt") in übereinstimmung mit dem 'Duden' 28 - als unerwartet oder zumindest überraschend vorzubereiten. Man kann sagen: Schriftsprachliche Texte werden versuchs- und stellenweise versprechsprachlicht. $\mathrm{Da}$ es auch einen sprachlichen Generationskonflikt gibt, gerade das sollte auch aus meinen Ausführungen deutlich werden.

\section{Jugendsprache und kritische Sprachwissenschaft}

Jugendsprache in der Gruppe ist experimentell, antikonventionell und tendenziell situationalisierend. Sprachkreativität ist das Vehikel dieser Eigenschaften, wobei Metaphorisierung und Hyperbolisierung die auffälligsten Eigenschaften sind. Dies insgesamt erlaubt es, von einem spezifischen 'Jugendton' zu sprechen. Dieser 'Jugendton' entfaltet sich erst einmal in der Gruppe, z.B. in der Gruppe gleichaltriger Schüler, die eine Klassenfreundschaft oder auch nur -kameradschaft verbindet. Der folgende Text thematisiert diesen Aspekt ${ }^{29}$ :

M. [in der schUle darf man sowiesO nich alles

[so Eng seh-n = + ich kEnn n paar lEUte'

[die $=+$ drEh-n in der schUle ganz AUf' LACHT +

$[$ un $=+$ sonst sin se Elgentlich + zlEmlich + rUhig + oder rUhiger $=++$

1. [in der klAsse drEh-n die AUf $=+\quad$ sonst würd ich AUch

M. โ ja-a

I. [schon SAg-n $=+$ was hEIßt - AUfdreh-n $=+$

M. $\quad\left[j a-A^{\prime}+\right.$ son bißchen rUmmOtz-n, ++ so wie hOpfi, LACHEN

I. $\quad \mathrm{jA}=$

[...]

H.-J. [wir drück-n in die Clty'+knUtsch-n Otz-n ma

H.-J. [rUnt-r' + LACHT

1. hAlt ma $=$ jEtzt + das mUB ich noch

H.-J. [ma hOr-n, / knUtsch-n Otze" ++ was is dAs $=+$

[wEiß nich, + grade Eb-n krelErt, LACHT +

[ $\mathrm{nE}$ also Otze is = + LACHEN + Otze is unbeschrEiblich, +

[. das is so was Ahnlich-s wie sUp-rkalifaliexperi 


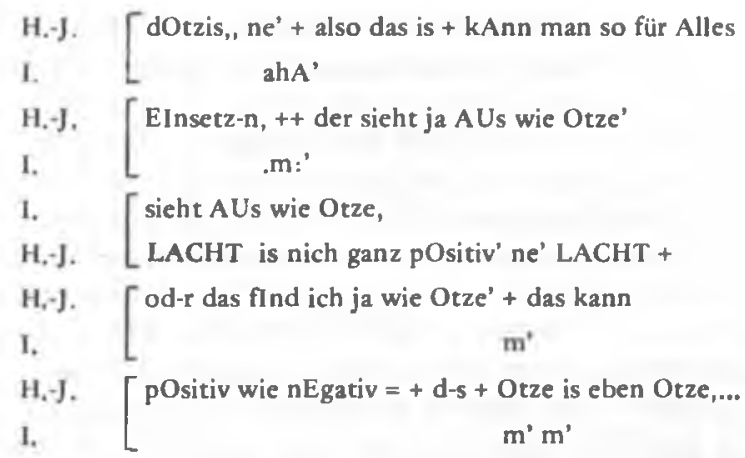

Dieser Text ist insofern ein Metatext, als er auf seine Weise den Zusammenhang von Peer-Group und Jugendton thematisiert. Allerdings läßt er sich nur bedingt auf eine Erklärungsattitude typisch wissenschaftlicher Provenienz ein. Auf eine Nachfrage hin wird aufdreben mit rummotzen $\mathrm{n}$ i c h t erklärt - muß man sagen; und als das schwebende $j a=$ des Interviewers diese Nichterklärung beklagt, wird auf einen Rummotzer, eben Hopfi, verwiesen. Sieh ihn dir an und du weißt, was Jugendton ist. Vielleicht sollte man noch hinzufügen, daß Jugendsprache sehr konkret ist.

Zu den zuvor angesprochenen Strukturmerkmalen noch einmal im einzelnen: Experimentell ist z.B., die Ellipse systematisch und gezielt zur Konstruktion von Grüßen und Glückwünschen einzusetzen: n'wunderschönen [Tag]! $n$ 'scbönes neues [Jahr]!; antikonventionell ist z. B., die sprachlichen Anreden im Umfeld von Mann/Herr und Frau/Dame ganz zu verlassen und durch Vorname und Artikel (das ist die Anja, ich bin der Thomas) bzw. "Exotismen" zu besetzen: Tussi, Gent, Fan, Freak; tendenziell situationalisierend ist, auf die semantische Kraft von Gesprächspartikeln zu setzen, auf Wörter im Gespräch, deren Bedeutung sich erst in der jeweiligen Situation erschließt. Dazu sei nochmals auf den vorstehenden Text verwiesen: "Otze ist eben Otze", das ist fast eine von den Jugendlichen für die Alten ("trau keinem über dreißig!") entworfene Formel für die Jugendsprache. Sie ist hermetisch und garantiert ungestörtes Innenleben. "Otze ist eben Otze!"

Auch in metaphorischen Prozessen, Beispiel aufdreben als 'rummotzen' und filmen als 'sich aufspielen', entfaltet sich jugendsprachliche Eigenart, vor allem aber in hyperbolischen Prozessen: etwas kann total niedlich, aber auch total beknackt sein; eine riesengroße Tussi ist sicherlich ein 'schönes' und, in unserer Sprache, möglicherweise ein 'zierliches' Mädchen, mit Sicherheit keine "übergroße Thusnelda". 
Was nicht dargelegt wurde, soll wenigstens zum Schluß noch Erwähnung finden: Jugendsprache, so könnte es scheinen, ist der Jungbrunnen der Erwachsenensprache. Daß es so einfach nicht ist, demonstriert ein Begriff, der bisher nicht eingeführt wurde: der Begriff der Stereotype. Die Kehrseite der in der Gruppe erworbenen und gepflegten Kreativität ist die der Stereotypisierung: Gesprächsphrasen werden z.B. dann zu Stereotypen, wenn sie zu b e l i e b i g e n Versatzstücken und Pausenfüllern verkommen, z.B. die gehäufte Verwendung von ich weiß nich: "Da war ich total/ ich weiß nich' / total frustriert". Oder auch der nur scheinbar interessante Einsatz des schriftsprachlichen et cetera (etc.), was in jugendlicher $\mathrm{Ge}$ sprächssprache sich als und so bzw. oder so artikuliert: "[...] 'nen ganzen Haufen / die kenn' ich gar nich' / auch deutsche Sachen und so". Die sich doch noch andeutende Denk- und Begriffsarbeit wird dann zerstört, wenn an die Stelle dieser Gesprächsphasen die flinken Entzückungs- und Beglaubigungswörter wie z.B. echt, toll, unbeimlich, tierisch treten. 30

Sprachprofilierung als Möglichkeit der jugendlichen Identitätsbildung ("innere Identität" ${ }^{31}$ ) soll heißen, daß das jugendliche Individuum in der Gruppe eine Ich-Änderung als Ich-Entfaltung und Hebung des Selbstwertgefühls erfährt. Das Individuum in der Gruppe macht die Erfahrung neuer Formen der Kommunikation und darauf bezogener neuer Kommunikationsmittel. Es wird jedoch ein Zeitpunkt kommen, da die jugendliche Gruppe einen eher retardierenden sprachlichen Einfluß ausübt. Darüber müßte innerhalb einer Theorie der Jugend und ihrer Entwicklungsphasen gehandelt werden. Sicher ist, daß die Jugend den Abschied von der Jugend und damit auch die Ablösung von der Jugendsprache selbst leisten muß, denn Jugend stellt ein Durchgangsstadium dar. Dagegen ist es Aufgabe der germanistischen Sprachwissenschaft, Bewertungskriterien für eine Kritik der Jugendsprache zu entwickeln. Dies ist insbesondere die Aufgabe einer kritischen Sprachwissenschaft, die, ich wiederhole mich, die Sprache i h r e r Zeit in zureichende Gedanken zu fassen hat. ${ }^{32}$ Damit wenigstens für die vielen Junggebliebenen unter uns die zeitliche Dimension der gegenwärtigen Jugendsprache etwas deutlicher wird, soll zum Abschluß noch einmal ein Jugendlicher zu Wort kommen: "Ich kann mich an die Beatles noch erinnern. Meine Mam kaufte gewöhnlich ihre Schallplatten."

\section{Anmerkungen}

Die vorangestellten Erläuterungen sind Teil meiner vor dem Vortrag verteilten Handreichungen. Sie sollen an dieser Stelle einen schnellen Oberblick gewähren. 
N. Chomsky: Aspekte der Syntax-Theorie. Frankfurt/M. 1969, 43.

2 S. Kierkegaard: Stadien auf des Lebens Weg. Düsseldorf, Köln 1958.

3 Vgl. u.a. B.U. Biere: Kindersprache, kindliche Kommunikation und Spracherwerb, in: ZGL 8.1980, $236 \mathrm{ff}$.

4 Zur Jugendforschung vgl. u.a.: L. v. Friedeburg (Hrsg.): Jugend in der modernen Gesellschaft. 6. Aufl. 1969; Zeitschrift für Pädagogik 25.1979, 659 - 830 (Heft 5), das dem Thema "Jugendprobleme - Jugendforschung" gewidmet ist; D. Baacke: Die 13-bis 18 jährigen. Einführung in Probleme des Jugendalters. 2. Aufl. 1979; vgl. auch Anm. 6.

5 G. Heinrich: Die Sprache der Jugend, in: Sprachwart 10.1960, Nr. 3, 41 f.; S.A. Wolf: Die Ische, die Brumme und der steile Zahn, in: Sprachwart 9. 1959, 165 f.; H. Markus: Zum Twen-Deutsch, in: ZDWF 18.1962, 151 - 159; S. Pape: Bemerkungen zur sog. Teenager- und Twensprache, in: Muttersprache 80.1970, 368 - 377; H. Küpper: Die deutsche schülersprache 1973/74, in: Wirkendes Wort 27.1977, 318 - 329; vgl. auch M. u. H. Küpper: Schülerdeutsch. Hamburg/Düsseldorf 1972; L. Ortner: Wortschatz der Pop-/Rockmusik. Düsseldorf 1981.

6 K.R. Allerbeck/L. Rosenmayr: Einführung in die Jugendsoziologie. Theorien, Methoden und empirische Materialien. Heidelberg 1976, 106.

7 Ebd., 110.

8 L. Rosenmayr: Jugend. 2. Aufl. Stuttgart 1976 (Handbuch der empirischen Sozialforschung. 6), 115 f., 276 f.; K.R. Allerbeck/L. Rosenmayr (Anm. 6), $99 \mathrm{f}$.

9 Gemeint sind R. Klappenbach/W. Steinitz (Hrsg.): Wb. der dt. Gegenwartssprache. Bd. 3. Berlin 1973; G. Drosdowski (Hrsg.): Das große Wb. der dt. Sprache. Bd. 3. Mannheim [usw.] 1977.

10 G. Wyneken: Was ist "Jugendkultur"? Offentl, Vortrag, gehalten am 30. Okt. 1913 [...], in: Grundschriften der dt. Jugendbewegung, hrsg. v. W. Kindt, Düsseldorf/Köln 1963, 116 - 128; H. Hartwig: Jugendkultur. Asthetische Praxis in der Pubertät, Reinbek 1980.

11 Allerbeck/Rosenmayr (Anm. 6), $100 \mathrm{f}$.

12 Vgl. Weigand/Hirt, Deutsches Wörterbuch. Bd. 1. Gießen 1909, 657 (Gryphius, Dornrose 51,5 ).

13 A. Hupe/H.-D. Schlawis: Zum Grußverhalten Jugendlicher. HauptseminarArbeit Braunschweig, Wintersemester 1979/80.

14 E. Goffman: Das Individuum im öffentlichen Austausch. Frankfurt/M. 1974, $113 \mathrm{f}$, i vgl, auch D. Hartmann: Begrüßungen und Begrüßungsrituale, in: ZGL 1.1973, 133 - 162.

15 G. Schank/G. Schoenthal: Gesprochene Sprache. Eine Einführung in Forschungsansätze und Analysemethoden. Tübingen 1976, 74.

16 F.J. Berens: Bemerkungen zur Dialogkonstituierung. In: F.J. Berens [u.a.] , Projekt Dialogstrukturen. München 1976, 31. 
17 H. Henne/H. Rehbock: Einführung in die Gesprächsanalyse. Berlin/New York 1979, 83.

18 Ebd., 182.

19 G. v. Wilpert: Sachwörterbuch der Literatur, 5. Aufl. 1969, 355.

20 Vgl. E. Henscheid: Ein scharmanter Bauer. Frankfurt/M. 1980.

21 Das Gespräch haben Petra Brockmann und Gabi Königsdorf im Rahmen ihrer Seminararbeit: "Die Welt der Auszubildenden und ihre Sprache" im Wintersemester 1979/80 geführt.

22 Vgl. H. Henne/H. Rehbock (Anm. 17), 182.

23 J. Rehbein: Sprechhandlungsaugmente. Zur Organisation der Hörersteuerung, in: H. Weydt (Hrsg.), Die (sic) Partikeln der deutschen Sprache, Berlin, New York 1979, $60 \mathrm{f}$.

24 Ebd., 65.

25 R. Klappenbach/W. Steinitz (Hrsg.): Wb. der dt. Gegenwartssprache, Bd. 4, Berlin 1977, 2607.

26 H.-P. Richter, in: Sounds. Platten 66 - 77. Frankfurt/M. 1979, 1338.

27 Sounds. Januar 1/1980, S. 4.

28 Duden. Rechtschreibung der dt. Sprache und der Fremdwörter. Mannheim [usw.] 1980, 29.

29 Das Textfragment entstammt einem Gruppeninterview mit 16jährigen, das ich am 7.1.1980 aufgenommen habe.

30 Vgl. G. Drosdowski/H. Henne: Tendenzen der dt. Gegenwartssprache, in: LGL, 2. Aufl. 1980, 630.

31 E.H. Erikson: Identifikation und Identität. In: Jugend in der modernen Gesellschaft. Hrsg. von L.v. Friedeburg. Köln 1976, 277. Vgl. auch E.H. Erikson: Jugend und Krise. Stuttgart 1970, $132 \mathrm{f}$.

32 Vgl. H. Henne: Fachidiome: Ober die eigene Zeit, studiert an der Sprache, in: Fachsprachen und Gemeinsprache. Jb. 1978 des IdS, Düsseldorf 1979, 302 316. 